Tecno Lógicas

ISSN 0123-7799

Vol. 17, No. 33, pp. 21-29

Julio- diciembre de 2014
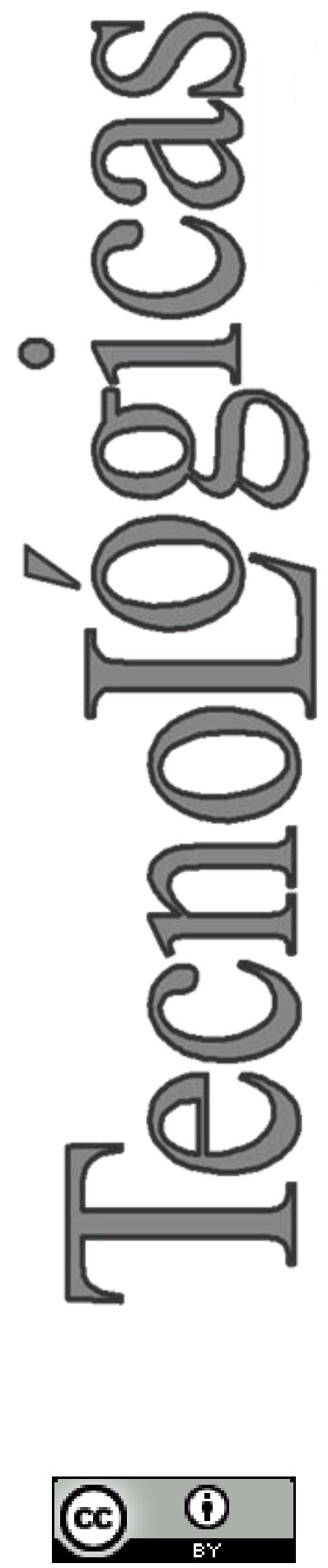

\section{Estimación automática de la medida de ocupación de playas mediante procesamiento de imágenes digitales}

\section{Automated estimation of occupancy measure on beaches using digital image processing}

Germán Sánchez-Torres ${ }^{1}$ y John A. Taborda-Giraldo

Recibido: 28 de enero de 2014,

Aceptado: 4 de abril de 2014

Cómo citar / How to cite

G. Sánchez-Torres y J. A. Taborda-Giraldo, "Estimación automática de la medida de ocupación de playas mediante procesamiento de imágenes digitales", Tecno Lógicas, vol. 17, no. 33, pp. 21-29, 2014.

1 Doctor en Ingeniería de Sistemas, Programa de Ingeniería de Sistemas, Facultad de Ingenierías, Universidad del Magdalena, Santa Marta-Colombia, gsanchez@unimagdalena.edu.co

2 Doctor en Ingeniería Electrónica, Programa de Ingeniería Electrónica, Facultad de Ingenierías, Universidad del Magdalena, Santa Marta-Colombia, jtaborda@unimagdalena.edu.co 


\section{Resumen}

Este trabajo describe una metodología para la estimación de densidad de ocupación de usuarios en playas mediante técnicas de procesamiento digital de imágenes. La estimación de la cantidad de usuarios en playas dedicadas al turismo constituye una métrica de interés que permite la cuantificación de diferentes factores que relacionan la calidad de la experiencia para el usuario y constituye un indicador relevante para la gestión integrada. El método propuesto posee dos componentes principales: el preprocesamiento y los procedimientos orientados para la estimación de densidad de ocupación. En el preprocesamiento de la imagen se aplica un método de corrección de color debido a que las múltiples variaciones de iluminación a través del día alteran la información de los objetos que se encuentran en la escena. Posteriormente, la estimación de la densidad inicia con la delimitación de la información referente al entorno de la imagen, mediante una imagen de referencia o máscara. Esta región obtenida es procesada identificando los usuarios mediante el análisis del gradiente de la intensidad en escalas de grises de la imagen. Los resultados experimentales muestran un error aproximado del $2,6 \%$, lo que sugiere que la medida estimada es adecuada como estimación de ocupación, las principales dificultades se centran en el tratamiento automático de las auto-oclusiones.

\section{Palabras clave}

Corrección de color, detección de bordes, estimación de densidad, procesamiento digital de imágenes, playas turísticas.

\section{Abstract}

In this paper we describe a methodology for occupancy measure on beaches based on digital image processing techniques. This metric constitute a measure of interest for quantification of factors relates to the quality of user experience and is an import indicator in integrated management field. The proposed method has two main components: the preprocessing and occupancy density estimation. In the preprocessing stage a color correction approach is applied in order to minimize the illumination variance effects over the objects in the scene. Subsequently, the estimation begins delimiting the information regarding the environment using a mask or reference image. Gray-level gradient analysis is applied to the resulted image after the mask appliance. The results show a $2.6 \%$ margin of error that suggests the measure is an occupancy measure indicative. The obtained error is related to the auto occlusion identification and processing.

\section{Keywords}

Color correction, edge detection, occupancy measure, digital image processing, tourist beaches. 


\section{INTRODUCCIÓN}

Actualmente, existe un interés creciente por sistemas que utilizan tecnología de visión asistidas por computadoras para el monitoreo de diferentes tipos de entornos. Este tipo de sistemas poseen diferentes objetivos que varían desde la seguridad, administración de recursos, publicidad, entre otros.

Los sistemas de estimación de multitudes son un ejemplo de la aplicación de esta tecnología [1]. Tradicionalmente, los enfoques de estimación de multitudes basados en visión por computador intentan detectar cada individuo $\mathrm{u}$ objeto en las imágenes para luego identificarlos [2]. Sin embargo, en los sistemas de conteo de multitudes la identificación individual de personas es una tarea computacionalmente demandante y está sujeta a imprecisiones debido a la dificultad de la separación de los objetos causada por la presencia de auto oclusiones.

Así, un enfoque general está orientado a la estimación de densidades de individuos, lo que sugiere una medida de la ocupación de un espacio físico sin el rigor de la determinación de la cantidad de los individuos. En general, una taxonomía de los sistemas de conteo de multitudes basado en imágenes puede dividirse en tres tipos de procedimientos [3]: detección individual de personas [2], [4], [5], agrupamiento de características visuales de la trayectoria [6], [7] y análisis basados en funciones de regresión [8].

Independientemente del tipo del método empleado, los sistemas de estimación de multitudes pueden ser incorporados en los sistemas de monitoreo en playas como herramienta para la estimación de la capacidad de carga. La capacidad de carga es una métrica que relaciona un espacio, un conjunto de usuarios y algunas medidas de calidad estimadas como resultado de la interacción de los usuarios y el espacio [9]. Formalmente, ésta métrica representa la cantidad de población que puede ser sopor- tada indefinidamente por un ecosistema sin destruirlo. En este sentido, la estimación de la cantidad de usuarios en playas dedicadas al turismo constituye una métrica de interés que permite la cuantificación de diferentes factores que relacionan la calidad de la experiencia para el usuario, adicionalmente, desde la perspectiva ambiental, constituye un indicador relevante para la gestión integrada de estos entornos [10].

Investigaciones como las de Jiménez et al. [11] y la de Botero et al. [12], consideran la densidad de ocupación de las playas como un factor fundamental en la planeación y gestión costera. Habitualmente, la medición de densidad de usuarios y capacidad de carga de playas turísticas se ha realizado de forma manual, a través de conteos en franjas determinadas por técnicas de campo. En [13] se presenta un estudio de vigilancia tecnológica al interior de un programa de monitoreo ambiental en playas turísticas, en el cual se hizo un diagnóstico de diversos procesos críticos, entre ellos, la estimación manual de la densidad de usuarios. La automatización de la estimación de la medida de ocupación de playas permite optimizar las labores de monitoreo, bajo los criterios de minimización de recursos y maximización de la calidad de los datos.

Este trabajo está orientado hacia el diseño de una metodología basada en procesamiento digital de imágenes que permita estimar una aproximación de la cantidad de usuarios presentes en una playa. El objetivo principal es la estimación de la densidad de uso de la playa, entendida como la cantidad de espacio utilizado por los usuarios y los elementos típicos que convergen en dicho entorno. Para la formulación de la metodología se tomó, como caso de estudio, imágenes de la playa El Rodadero en Santa Marta, Colombia. Los resultados indican que la métrica resulta adecuada como aproximación del nivel de ocupación de una playa y permite la automatización de este procedimiento. 


\section{MATERIALES Y MÉTODOS}

El método propuesto posee dos componentes principales: el preprocesamiento y los procedimientos orientados para la estimación de densidad de ocupación. En el preprocesamiento de la imagen se aplica un método de corrección de color debido a que las múltiples variaciones de iluminación a través del día alteran la información de los objetos que se encuentran en la escena. Posteriormente, la estimación de la densidad inicia con la delimitación de la información referente al entorno de la imagen, mediante una imagen de referencia o máscara. Sobre este segmento de imagen se aplica un procedimiento para la eliminación de información de objetos conocidos. Los objetos conocidos los conforman elementos con características fijas de color que no son relevantes para el análisis. Este grupo de objetos puede variar de acuerdo con el entorno y es una etapa de configuración por parte del usuario. La Fig. 1 muestra el diagrama del procedimiento.

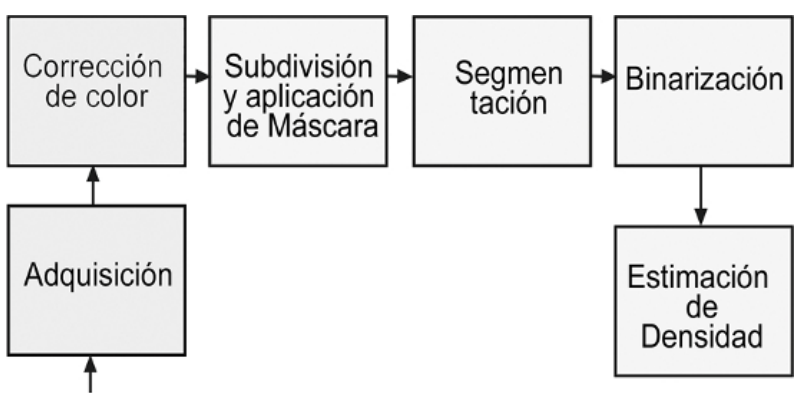

Fig. 1. Esquema de la metodología propuesta. Fuente: Autores

\subsection{Adquisición de imágenes}

El diseño del método está orientado a la utilización de dispositivos tradicionales de captura. Las imágenes se adquieren en formato RGB24 con 2592x1944 pixeles y una resolución horizontal y vertical de 314 pp y almacenadas en formato PNG (Portable Network Graphics). Para este trabajo la zona estudiada corresponde a la Bahía del Rodadero, con coordenadas geográficas $11^{\circ} 12^{\prime} 18,9^{\prime \prime} \mathrm{N}-74^{\circ} 13^{\prime} 41,6^{\prime \prime} \mathrm{W}$ con una longitud de $1186 \mathrm{~m}$ y un área aproximada de $55683 \mathrm{~m}^{2}$. La Fig. 2 muestra ejemplos del sitio descrito.

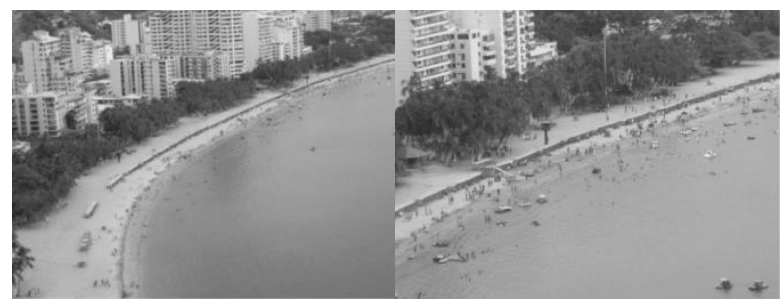

Fig. 2. Ejemplos de las imágenes adquiridas. Fuente: Autores

\subsection{Preprocesamiento de la imagen: corrección de color}

La corrección de color, la eliminación de ruido y el mejoramiento de bordes son procedimientos típicos que se aplican en esta fase de un proyecto basado en procesamiento digital de imágenes. La corrección de color en imágenes es necesaria debido a que los objetos podrían alterar su posición en el espacio de color causado por las variaciones de la intensidad de la luz del entorno durante el transcurso del día. Para esto se debe considerar que las características de color de la imagen adquirida mediante una cámara dependa de tres factores, a saber, el contenido de la escena adquirida, la iluminación incidente y las características del dispositivo de adquisición, estos últimos factores afectan directamente la representación del color de los objetos. Debido a que las imágenes son adquiridas con el mismo dispositivo y configuración, el método de corrección de color está orientado a disminuir la iluminación incidente.

En un modelo ideal la formación de la imagen es descrita por (Graham et al. [14]):

$p_{k}^{x}=\int_{w} E(\lambda) S^{k}(\lambda) R^{k}(\lambda) d \lambda$

Donde, $p_{k}^{x}$ es la respuesta del sensor $k$ en el pixel $x$. $E(\lambda)$ es la distribución espec- 
tral de la potencia de la iluminación incidente, $S^{k}(\lambda)$ la función de reflectancia de la superficie, $R^{k}(\lambda)$ es la sensibilidad de la cámara a la longitud de onda $\lambda$ del espectro visible $w$. Los algoritmos de corrección de color o constancia de color asumen una única fuente de luz y estiman la distribución espectral de la iluminación $E(\lambda)$. Se utilizó el método propuesto en [15] para la constancia de color usando características de bajo nivel denominado Grey-Edge, el cual está basado en las derivadas de orden superior de la imagen. La técnica puede ser expresada como:

$\left(\left|\frac{\partial^{n} i^{\sigma}(x)}{\partial x^{n}}\right|^{p} d x\right)^{\frac{1}{p}}=k l^{n, p, \sigma}$

Donde, $n$ es el orden de la derivada, $p$ es la norma Minkowski y $\sigma$ es el parámetro de un filtro gaussiano de suavidad de la imagen $i$. Esto permite disminuir el efecto de las variaciones de iluminación durante el día, como se evidencia en la Fig. 3, imágenes tomadas en diferentes horas resultan en tonalidades similares después de la corrección.

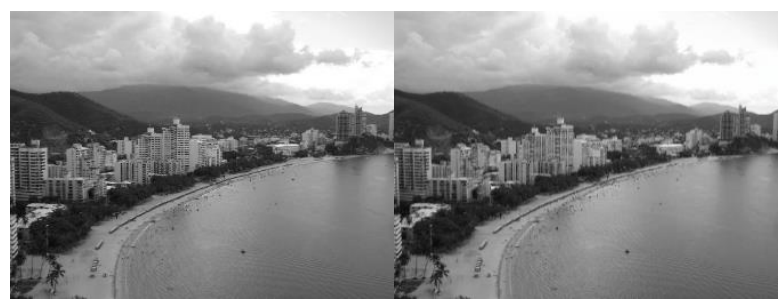

a)

b)

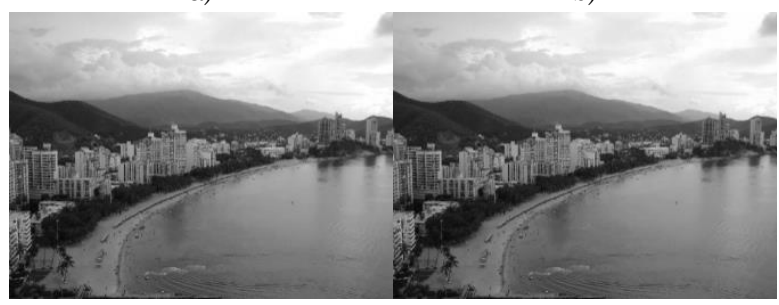

c)

d)

Fig. 3. Aplicación del procedimiento de corrección de color, a y c) imágenes originales, b y d) resultado de la corrección. Fuente: Autores

Finalmente, se aplica un procedimiento de realce de bordes o Image Sharpening. El objetivo es mejorar los detalles determina- dos por las líneas estructurales que describen elementos en la imagen. Esto es logrado mediante la atenuación de las frecuencias bajas manteniendo las frecuencias altas de la transformada de Fourier sin modificaciones significativas. De tal forma que, dado una función de transferencia de un filtro pasa bajas $H_{L P}(u, v)$, el funcional de transferencia pasa altas está determinado por $H_{H P}(u, v)=1-H_{L P}(u, v)$, por lo que se definió que $H(u, v)=1-e^{-D^{2}(u, v) / 2 D_{0}^{2}}$.

\subsection{Subdivisión de la imagen y aplicación de la máscara}

Debido a que las imágenes fueron desde posiciones altas, estas incluyen información poco relevante para el análisis. Por lo tanto, las imágenes adquiridas fueron recortadas mediante la aplicación de una máscara. Las máscaras deben ser definidas una vez al inicio y hace parte de la configuración del sistema (ver Fig. 4a y 4b).

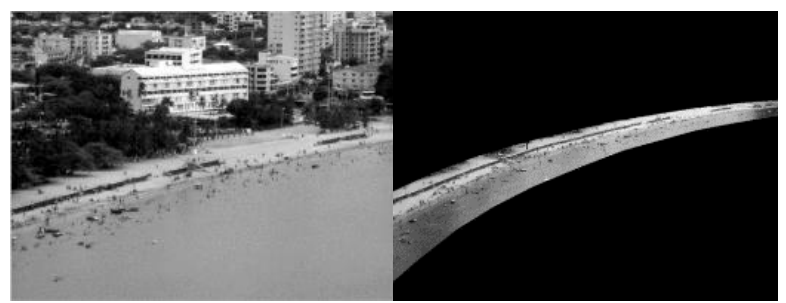

a)

b)

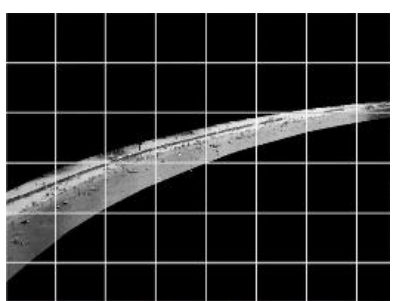

c)

Fig. 4. Imagen de escala de grises y su máscara correspondiente y c) ejemplo de subdivisión de imagen. Fuente: Autores

Posteriormente, se realizó una subdivisión del espacio de estudio, para disminuir la complejidad del procesamiento y generar unidades más pequeñas de menor tamaño y cómputo más rápido. La Fig. 4c, muestra gráficamente la subdivisión espacial obtenida. El cómputo del método es realizado 
mediante el análisis independientemente de cada una de las partes en la subdivisión.

\subsection{Segmentación y binarización}

El interés principal en esta etapa es encontrar la segmentación de los diversos elementos que ocupan espacio dentro de la escena y está direccionada hacia la determinación de los espacios ocupados en la región descrita en la imagen.

Existen diferentes técnicas para realizar la segmentación de objetos en una imagen y su objetivo es descomponer la imagen en los objetos que la conforman. En general, los métodos de segmentación pueden agrupase en globales o locales. La segmentación local es adecuada cuando la escena presenta variaciones en la iluminación. Sin embargo, los métodos clásicos utilizan un valor de referencia o umbral para realizar la segmentación globalmente. La correcta selección del umbral es dependiente del tipo de escena y de las características propias de los objetos en la imagen, es decir es dependiente del problema. Para la selección del método de segmentación este trabajo se basó en los estudios realizados por [16] y [17], que muestran una comparativa de los métodos de segmentación y concluyen que el operador prewitt constituye uno de los mejores algoritmos para detectar bordes verticales [16] y a su vez uno de los menos costosos computacionalmente [17].

El operador Prewitt asume un peso similar en el gradiente vertical, horizontal y diagonal. En las imágenes bidimensionales las derivadas constituyen un vector en las direcciones de las máximas variaciones locales. Estos valores son proporcionales al nivel de la variación, en cuanto mayor variación se encuentre en una localidad de la imagen mayor será el modulo del gradiente. En este contexto los niveles de variación están referidos a las variaciones en la escala de grises que representa el contenido de la imagen. Los gradientes pueden definirse como: $\nabla f(x, y)=\left[\begin{array}{l}\frac{\partial f(x, y)}{\partial x} \\ \frac{\partial f(x, y)}{\partial y}\end{array}\right]$

Y su magnitud está definida como:

$M(\nabla f(x, y))=\sqrt{\left(\left(\frac{\partial f(x, y)}{\partial x}\right)^{2}+\left(\frac{\partial f(x, y)}{\partial y}\right)^{2}\right)}$

La generación de los gradientes se realiza en la localidad de cada pixel dentro de la imagen. Esta localidad es analizada mediante la aplicación de núcleo de transformación de la primera derivada en las direcciones ortogonales que representan los ejes $x$ y $y$. Los núcleos fueron definidos como:

$\frac{1}{3}\left[\begin{array}{lll}-1 & 0 & 1 \\ -1 & 0 & 1 \\ -1 & 0 & 1\end{array}\right] \quad \frac{1}{3}\left[\begin{array}{ccc}-1 & -1 & -1 \\ 0 & 0 & 0 \\ 1 & 1 & 1\end{array}\right]$

Este operador es aplicado mediante la convolución descrita en (6).

$O(i, j)=\sum_{k=1}^{3} \sum_{l=1}^{3} I(i+k-2, j+l-2) K(k, l)$

Donde, $O(i, j)$ es el valor del pixel $(i, j)$ de la imagen estimada, $I(i, j)$ es el pixel de la imagen inicial y $K(k, l)$ es el valor del kernel de convolución.

El efecto de la aplicación de la estimación del gradiente a la Fig. 5a puede observarse en la Fig. 5b. Una vez obtenidos los gradientes, estos son utilizados para generar una primera aproximación del espacio ocupado por cada objeto descrito en la imagen gradiente. Se aplicó un operador de relleno con conectividad de cuatro puntos. El operador de relleno cierra los contornos que los gradientes que pueden ser descritos mediante una conectividad de 4 puntos.

Sin embargo, este relleno no suele ser completamente demarcado. Esta mejora del contraste del interior de los objetos es generado mediante la aplicación de un 
operador morfológico con un elemento estructural de máscara (7), y posteriormente binarizada (ver Fig. 5c).

$$
\operatorname{mask}=\left[\begin{array}{ll}
1 & 1 \\
1 & 1
\end{array}\right]
$$
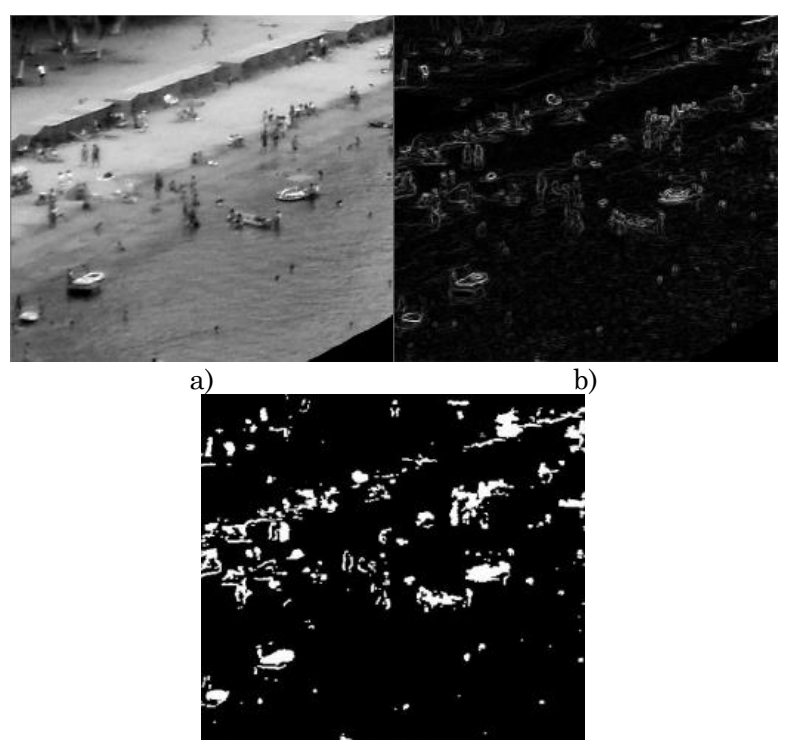

c)

Fig. 5. a) Segmento para el cual se aplicará el proceso final, b) resultado de la estimación del gradiente y c) imagen umbralizada. Fuente: Autores

Finalmente, la medida de densidad de ocupación está determinada por:

$d_{o}{ }^{i}=\frac{B_{i}}{R_{i}}$

Donde, $B_{i}$ es el conjunto de pixeles de la imagen binarizada del segmento $i$ de la subdivisiones y $R_{i}$ el conjunto de pixeles de la región $i$ que se encuentra dentro de la máscara. Por lo tanto, la medida final es:

$D_{o}=\frac{\sum_{i=1}^{n} d_{0}^{i}}{n}$

\section{RESULTADOS}

Se definió la métrica de error $\varepsilon$ basada en la diferencia entre una imagen segmentada por el método propuesto $\operatorname{Im}_{i}$ y una obtenida a través de la substracción de una imagen de fondo obtenida mediante intervención manual $I m_{\text {fondo }}$ y la imagen analizada $\operatorname{Im}_{i}$, ver (10).

$$
\begin{aligned}
& \varepsilon=\left|\frac{\operatorname{Im}_{\text {ref }}-I m_{i}}{I m_{i}}\right| \\
& =\left|\frac{\left(\text { binarizada }_{\left.\left(\operatorname{Im}_{\text {fondo }}-I m_{i}\right)-I m_{i}\right)} \mid\right.}{\operatorname{Im}_{i}}\right|
\end{aligned}
$$

La Fig. 6 ejemplifica una imagen $\operatorname{Im}_{i} \mathrm{y}$ su correspondiente $\operatorname{Im}_{\text {fondo. }}$

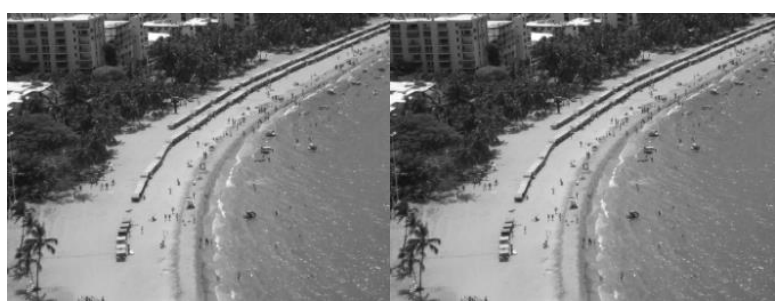

a)
Fig. 6. a) Imagen $I m_{i}$ y b) el fondo correspondiente $I m_{\text {fondo }}$. Fuente: Autores

Para evaluar el método propuesto fue necesario estimar el valor correcto del umbral del proceso de binarización. Para esto, se analizó el comportamiento del error mediante la variación del umbral. Se estimó el nivel de ocupación de las imágenes y la imagen generada en cada prueba, con valores de umbral variando desde 0,05 hasta 0,6 .

Los resultados obtenidos se muestran en la Fig. 7a. La medida del error reportada es estimada como la diferencia de los porcentajes de ocupación de la imagen de referencia y la obtenida con el método propuesto en cada prueba. Los valores menores de error se encuentran en el rango de 0,3 y 0,4 , por lo tanto, el valor del umbral se estableció en 0,35. El histograma de los resultados de los errores encontrados con esta parametrización, aplicada a un conjunto de 400 imágenes se encuentra en la Fig. 7b. El comportamiento del error varía en un rango de $0,002 \%$ y $5,25 \%$, con un promedio de $2,68 \%$ y la desviación típica de 1,75 para el conjunto de imágenes utilizadas. 


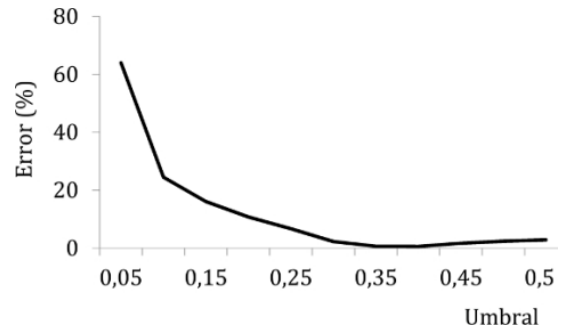

a)

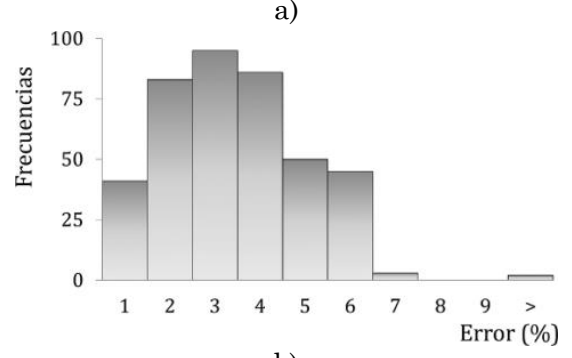

b)

Fig. 7. a) Comportamiento del error mediante la variación del valor del umbral de binarización y b) histograma del error para el conjunto de 400 imágenes. Fuente: Autores

La Fig. 8 muestra un ejemplo de una imagen sintética, a la cual se le añadió información correspondiente al 5\%, 10\% y 15\% de la imagen inicial (ver Fig. 8a, 8c y 8e). Las estimaciones de la ocupación por el método propuesto corresponden al $7,8 \%$, $11,75 \%$ y $16,2 \%$ respectivamente, y se muestran en las Fig. 8b, 8d y 8f.

\section{CONCLUSIONES}

Un análisis preliminar, indica que la cuantificación de la métrica de carga de playas en el sentido estricto de la definición de los autores, no es una tarea trivial debido a la diferenciaron de los actores. Esta clasificación está inmersa en la definición de la métrica en el sentido en que cada uno de estos incorpora una carga ambiental desde diferentes escenarios.

El desarrollo de este trabajo intenta una aproximación a esta métrica orientada a la cuantificación del área ocupada como un indicador de influencia en los parámetros de calidad desde la perspectiva turística. Las oclusiones generadas por la presencia de vegetación, dificulta la estimación de la métrica en esas regiones. Trabajos futu- ros estarán orientados a mejorar la estimación del área ocupada por los elementos presentes en estas zonas que no corresponden a personas. Un análisis previo sobre el espacio de color podría mejorar dicha estimación.

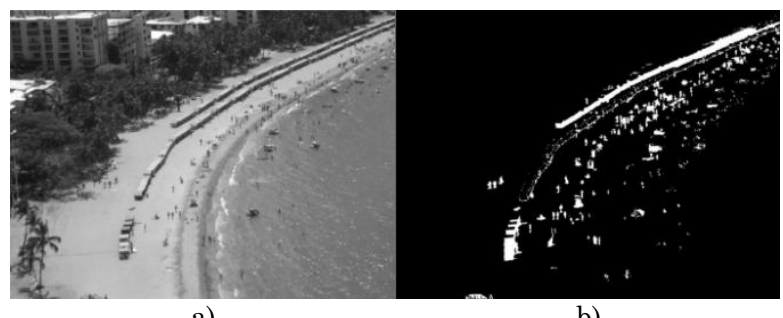

a)

b)

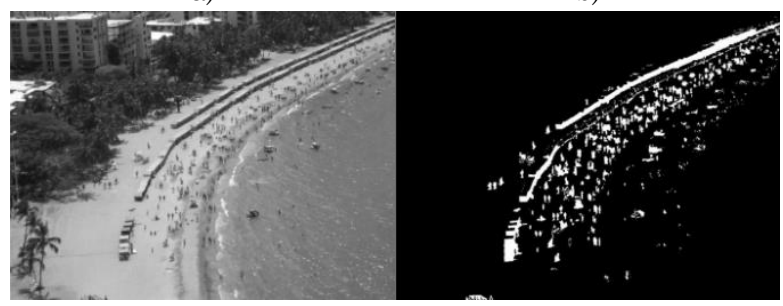

c)

d)

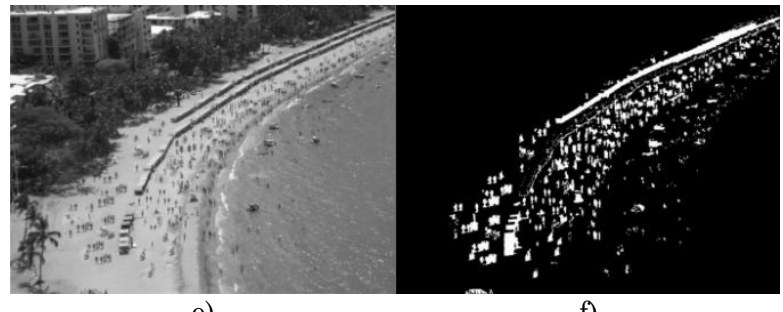

e)

f)

Fig. 8. Imágenes sintéticas: a) imagen inicial, c) inicial más $5 \%$ y d) inicial más 10\% de información, b), d) y f) los resultados del método propuesto, respectivamente. Fuente: Autores

\section{REFERENCIAS}

[1] H. Su, H. Yang, and S. Zheng, "The Large-Scale Crowd Density Estimation Based on Effective Region," Lect. Notes Comput. Sci., vol. 6494, pp. 302-313, 2011.

[2] C. H. Chen, T. Y. Chen, D. J. Wang, and T. J. Chen, "A cost-effective people-counter for a crowd of moving people based on two-stage segmentation," J. Inf. Hiding Multimed. Signal Process., vol. 3, no. 1, pp. 1223, 2012.

[3] S. Yoshinaga, A. Shimada, and R. Taniguchi, "Realtime people counting using blob descriptor," Procedia Soc. Behav. Sci., vol. 2, no. 1, pp. 143-152, Jan. 2010.

[4] P. Negri, N. Goussies, and P. Lotito, "Detecting pedestrians on a Movement Feature Space," Pattern Recognit., vol. 47, no. 1, pp. 56-71, Jan. 2014.

[5] G. Sen, L. Wei, and Y. H. Ping, "Counting people in crowd open scene based on grey level dependence matrix," in 2009 International Conference on Information and Automation, 2009, pp. 228-231. 
[6] G. Antonini and J. P. Thiran, "Counting Pedestrians in Video Sequences Using Trajectory Clustering," IEEE Trans. Circuits Syst. Video Technol., vol. 16, no. 8, pp. 1008-1020, Aug. 2006.

[7] A. B. Chan and N. Vasconcelos, "Privacy preserving crowd monitoring: Counting people without people models or tracking," in 2008 IEEE Conference on Computer Vision and Pattern Recognition, 2008, pp. 17.

[8] N. Hussain, H. S. M. Yatim, N. L. Hussain, J. L. S. Yan, and F. Haron, "CDES: A pixel-based crowd density estimation system for Masjid al-Haram," Saf. Sci., vol. 49, no. 6, pp. 824-833, Jul. 2011.

[9] R. E. Manning and S. R. Lawson, "Carrying capacity as 'Informed Judgment': the values of science and the science of values.," Environ. Manage., vol. 30, no. 2, pp. 157-68, Aug. 2002.

[10] C. Botero, Y. Hurtado, J. González, M. Ojeda, and L. Díaz, "Metodología de cálculo de la capacidad de carga turística como herramienta para la gestión ambiental y su aplicación a cinco playas del Caribe Colombiano," Gestión y Ambiente, vol. 11, no. 3, pp. 17-23, 2008.

[11] J. A. Jiménez, A. Osorio, I. Marino-Tapia, M. Davidson, R. Medina, A. Kroon, R. Archetti, P.
Ciavola, and S. G. J. Aarnikhof, "Beach recreation planning using video-derived coastal state indicators," Coast. Eng., vol. 54, no. 6-7, pp. 507-521, Jun. 2007.

[12] C. Botero, C. Pereira, and E. Escudero, "Informe del Programa de investigación en Calidad Ambiental de Playas Turísticas (CAPT) en el Caribe Norte Colombiano 2010-2013," 2010.

[13] J. A. Taborda, C. Botero, G. Sánchez, and L. Camargo, "Innovación tecnológica en el programa de calidad ambiental de playas turísticas del Caribe Norte Colombiano: diagnóstico de problemas y soluciones," in II CAPT 2013, 2013, pp. 71-78.

[14] D. H. Foster, "Color constancy," Vision Res., vol. 51, no. 7, pp. 674-700, Apr. 2011.

[15] J. van de Weijer, T. Gevers, and A. Gijsenij, "EdgeBased Color Constancy," IEEE Trans. Image Process., vol. 16, no. 9, pp. 2207-2214, Sep. 2007.

[16] S. Al-amri, N. Kalyankar, and S. Khamitkar, "Image segmentation by using edge detection," Int. J. Comput. Sci. Eng., vol. 02, no. 03, pp. 804-807, 2010.

[17] M. Sridevi and C. Mala, "A Survey on Monochrome Image Segmentation Methods," Procedia Technol., vol. 6, pp. 548-555, Jan. 2012. 